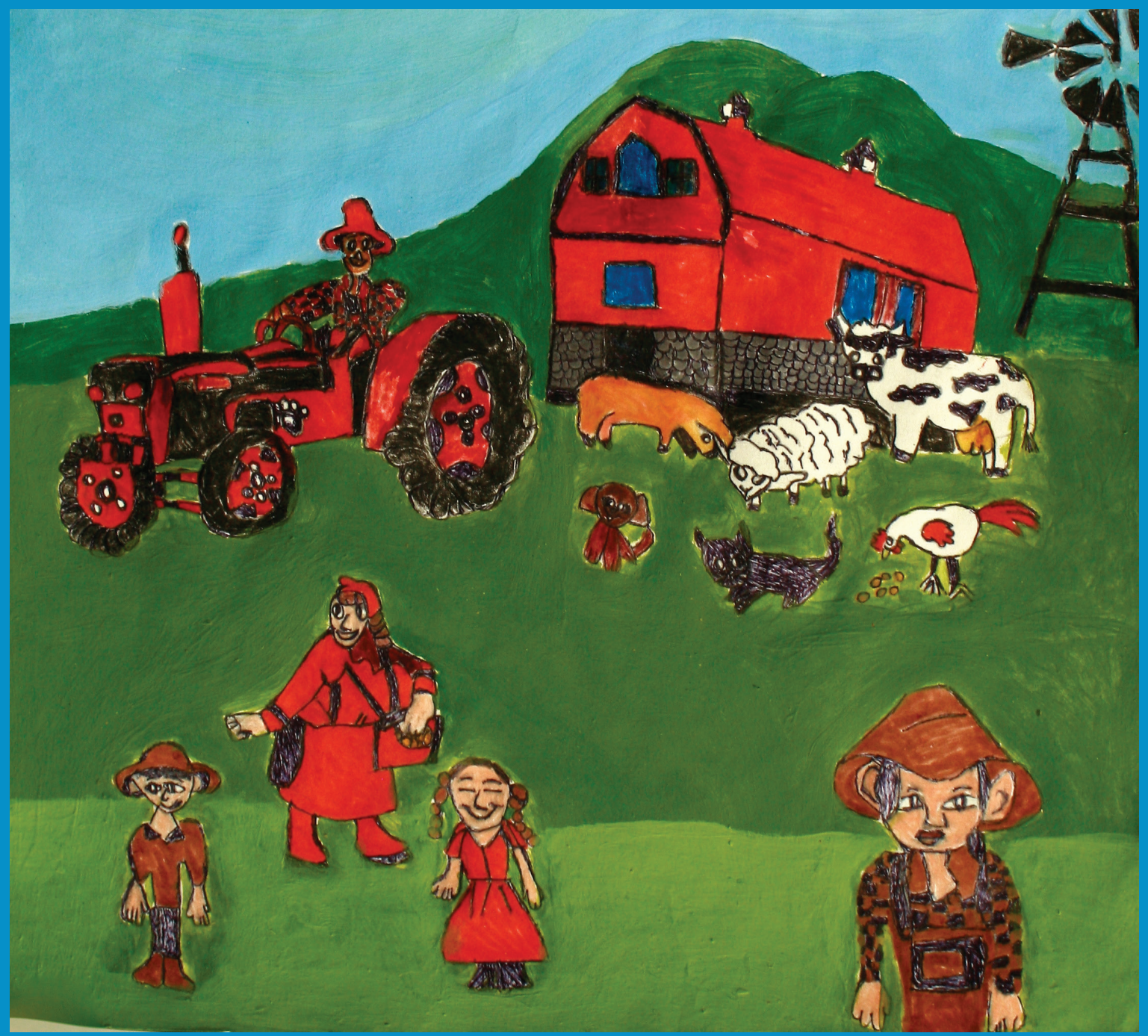

Victor Hugo Guerrero - Arte sin Fronteras - Programa Talentos Especiales

Adriana del Pilar Talero Parra.

Terapeuta Ocupacional.

Especialista en Gerencia Salud Ocupacional.

Magíster en Prevención de Riesgos Laborales.

Profesora Programa de Terapia Ocupacional.

Universidad del Rosario.

ataleroa@yahoo.com

Lizeth Dayana Mahecha.

Fisioterapeuta.

Terapeuta Ocupacional en formación.

Universidad del Rosario.

lizmahecha_g@hotmail.com

Nathalia Cubillos.

Terapeuta Ocupacional en formación.

Universidad del Rosario.

cubillos.nathalia@urosario.edu.co 


\title{
ACCESO DE PERSONAS CON DISCAPACIDAD A LA FORMACIÓN SUPERIOR DESDE LA PERSPECTIVA DE TERAPIA OCUPACIONAL
}

\section{Access to higher education to people with disability from the perspective of Occupational Therapy}

Fecha de recepción: 8 de mayo de 2014 - Fecha de aprobación: 1 de diciembre de 2014

\author{
RESUMEN
}

Según la OMS el acceso a los servicios básicos de formación en personas con discapacidad se ve ampliamente limitado, impactando negativamente en los procesos de inclusión laboral. Se propuso el objetivo de identificar la evidencia científica relacionada con el acceso a la formación superior de personas con discapacidad. Se realizó la búsqueda de evidencia en las bases de datos Labordoc, Ebsco, Scielo y Lilacs, a través de los tesauros "Discapacidad", "Competencias laborales", "Profesional", "Formación", "Tecnológico", "Técnico", "Educación superior" "estudio"y "Terapia Ocupacional", realizando con estos 10 combinaciones mediante el uso del boléano "AND". Los análisis fueron realizados mediante el establecimiento de frecuencias para variables como país, tipo de estudio, idioma y periodo de publicación. Se encontró un total de 20 artículos, en donde la mayor publicación fue Labordoc con el 45\%, el periodo de tiempo con más publicaciones es entre 2009-2013 con un 45\%, el idioma con más evidencia fue el inglés con el 60\%, Brasil y Colombia fueron los países con mayor número de publicaciones, cada uno con un $15 \%$. En el marco legislativo en discapacidad, se hacen evidentes aún las brechas entre la normatividad y la realidad, especialmente en la ejecución e implementación de programas, así como la falta de participación de diversas profesiones, por lo que a partir de este análisis, se hace extensiva la invitación a investigar y publicar artículos con relación a la formación superior desde cada uno de los enfoques de formación.

PALABRAS CLAVE

Discapacidad, formación superior, inclusión laboral, Terapia Ocupacional.

\begin{abstract}
According to WHO access to basic training in people with disabilities is greatly limited negative impact on the processes of labor inclusion. To identify the scientific evidence related the access to higher education for people with disabilities. The search for evidence on the basis of Labordoc , Ebsco , Lilacs and SciELO data was conducted through thesauri "Disability" , "Skills ", " Professional ", "Training ", " Technology ", " Technical " "Higher Education " and "Occupational Therapy" , performing with these 10 combinations using the boolean "AND". Analyses were performed by setting frequencies for variables such as country, study type, language and publication period. A total of 20 items, where most public Labordoc with $45 \%$, was found the time most published between $2009-2013$ is $45 \%$, most evidently language was English with $60 \%$, Brazil and Colombia were the countries with the highest number of publications , each with $15 \%$. Despite major advances in the legislative framework on disability, are evident even gaps between norms and reality, especially in the execution and implementation of programs and the lack of participation of various professions, so from this analysis is extended the invitation to research and publish articles regarding the top from each training approaches training.
\end{abstract}

KEY WORDS

Disability, higher formation, labor inclusion, Ocupacional Therapy. 
borales y de saneamiento de las personas con discapacidad, lo cual afectan considerablemente su calidad de vida.

De acuerdo a lo expuesto anteriormente, es importante resaltar que el acceso al mercado laboral formal depende del nivel educativo de las personas principalmente. Con base en lo anterior, se ha establecido por parte del DANE que de las cifras de población registrada para el año 2010, aproximadamente el 1.25\% corresponde a personas con discapacidad que han realizado estudios técnicos y tecnológicos, el $1.21 \%$ estudios Universitarios y el $0.23 \%$ estudios de posgrado, lo cual deja expuesto las bajas tasas de personas con discapacidad que acceden a la educación superior en el país (Departamento Nacional de Estadística, 2005).

De acuerdo a la Constitución Política Colombiana, la atención educativa de las personas con discapacidad es una obligación del estado y por ende se debe favorecer que las entidades educativas sean más inclusivas que exclusivas, con el objeto que no se vean afectados los procesos de escolarización y con ello la calidad de vida de las personas con discapacidad, y sus familias (Senado de la República de Colombia,1991), puesto que cada vez, se van volviendo aún más dependientes de sus cuidadores, lo que restringe su participación activa en la sociedad y altera su desempeño ocupacional en cada una de las áreas.

Para efectos de este estudio se tiene en cuenta una de las instituciones más representativas en Colombia en cuanto a educación como lo es el Servicio Nacional de Aprendizaje (SENA), con el cual se definen los tipos de educación superior existentes para Colombia, siendo estos la formación técnica y tecnológica.

La formación técnica busca la adquisición y desarrollo de competencias motrices, cognitivas, socio-afectivas y comunicativas para la realización de actividades determinadas y la solución a problemas en un rango definido, a través de la utilización de instrumentos y técnicas definidas para obtener resultados concretos. La formación tecnológica a su vez, busca el desarrollo de competencias relacionadas con la solución estratégica de problemas, la coordinación y supervisión de actividades, gestión de proyectos productivos y organización y manejo de recursos. Por último, la formación profesional implica el dominio operacional e instrumental de una ocupación a través de la apropiación de un saber técnico y tecnológico integrado, para plantear y solucionar creativamente los problemas de manera eficaz y adaptarse a los cambios constantes de la productividad (Servicio Nacional de Aprendizaje, sf).

Las personas con discapacidad para llegar a ser competentes y cumplir un rol laboral requieren de procesos de escolarización continuos, lo cual no se ve evidenciado durante el quehacer del terapeuta ocupacional en programas que promueven la inclusión laboral. Por lo tanto, el presente análisis bibliométrico se enfoca en conocer la evidencia científica en relación a la formación superior de personas con discapacidad y como ésta influye en el acceso a la fuerza laboral del país, lo cual representa procesos de independencia, autonomía y ganancias significativas en términos de desempeño ocupacional de la persona. 


\section{Fase 1: Tema a analizar.}

En la primera fase para la realización del análisis bibliométrico se tuvo en cuenta la selección del tema a analizar, bajo el contexto del área de práctica del programa de terapia ocupacional de la Universidad del Rosario en la Fundación Teletón Colombia, sede Bogotá, en la cual uno de los programas llevados a cabo, es la inclusión laboral para personas con discapacidad física o motora, sensorial, intelectual y psicosocial, en donde se ha puesto en evidencia la falta de formación superior de los usuarios que acuden, y con ello las dificultades de acceso al mercado laboral del país, siendo esto una limitación para la participación en las áreas de la ocupación especialmente las relacionadas con el trabajo, que a su vez afectan los patrones de ejecución y los contextos en los cuales está inmerso el individuo, alterando de manera significativa su desempeño ocupacional. El tema seleccionado corresponde a la formación técnica, tecnológica y profesional de personas con discapacidad.

\section{Fase 2: Selección de criterios de búsqueda}

Para la realización del presente análisis bibliometrico se establecieron criterios de búsqueda en los cuales se incluyeron: las bases de datos EBSCO, LILACS, SCIELO y OIT específicamente en su base de datos LABORDOC, los tesauros "Discapacidad", "competencias", "formación", "terapia ocupacional". "profesional”, "técnico", "tecnológico", "competencias laborales", "estudio” y "educación superior”, mediante el empleo del boléano "AND”, publicados entre los años 2000 al 2013 en idiomas inglés y español.

\section{Fase 3: Búsqueda de evidencia}

Se realizó la búsqueda de evidencia en las bases de datos anteriormente mencionadas por medio de los tesauros y boléanos establecidos como criterios de búsqueda, formando con ellos las combinaciones que a continuación se relacionan: "Formation" And "Technological" And "Disability" / "Competences" And "Technological" And "Disability" / "Formation" And "Technical" And "Disability" / "Formation" And "Professional" And "Disability" / "Work Formation" And "Disability" / "Competences" And "Professional" And "Disability" / "Work Competences" And "Disability" / "Higher Education" And "Disability" / "Occupational Therapy" And "Formation" And "Disability"

Las anteriores combinaciones se utilizaron en cada una de las bases de datos con los criterios de año e idioma.

\section{Fase 4: Consolidación de información}

Los resultados de la búsqueda que se realizó en cada una de las bases de datos fueron consolidados en un archivo Excel en las cuales se realizó una tabla que contenía las siguientes categorías: Base de datos, combinación, nombre de artículo por combinación, año, autor, tipo de estudio, resumen, introducción y conclusiones del mismo.
Para el anterior procedimiento y con el objeto de excluir artículos de información no relevante para el presente estudio, se realizaron tres filtros, siendo el primero de ellos por nombre del artículo en el cual se excluyeron aquellos cuyo nombre no proporcionaba una idea general en relación al tema de investigación. Una vez realizado esto se procedió al segundo filtro, el cual corresponde al abstract de cada uno de los documentos anteriormente seleccionados, si este no proporcionaba información relacionada al tema de investigación, era excluido. Por último, se realizó el tercer filtro correspondiente al contenido de cada uno de los documentos y que tenían relevancia con el objeto de estudio.

Fase 5: Estudio de artículos

En la última fase se realizó la lectura completa y detallada de cada uno de los artículos seleccionados tras el tercer filtro, se analizó cada uno de los documentos en su contenido para establecer los resultados relacionados con el objetivo del estudio.

\section{RESULTADOS}

En relación a la búsqueda realizada durante la primera fase, se encontró un total de 736 artículos para las 10 combinaciones establecidas anteriormente y en las cuatro bases de datos LILACS, EBSCO, SCIELO y LABORDOC, siendo esta última la que mayor número de artículos proporciono (ver tabla 1).

Tabla 1. Publicaciones identificados para cada base de datos

\begin{tabular}{|l|c|c|}
\hline \multicolumn{1}{|c|}{ Base de datos } & $\mathbf{N}^{\circ}$ de artículos & $\%$ \\
\hline LABORDOC & 410 & $56 \%$ \\
\hline EBSCO & 231 & $31 \%$ \\
\hline SCIELO & 52 & $7 \%$ \\
\hline LILACS & 43 & $6 \%$ \\
\hline Total & $\mathbf{7 3 6}$ & $\mathbf{1 0 0} \%$ \\
\hline
\end{tabular}

Del total de artículos encontrados, al realizar el primer filtro en relación al título de los mismos, se obtuvo un total de 121 artículos, siendo la combinación con mayor publicaciones "Higher Education" And "Disability" (Tabla 2)

Luego de la realización de este primer filtro, se continuó con el filtro relacionado con el abstract de cada una de las publicaciones en relación a los criterios de búsqueda, quedando un total de 53 artículos (Tabla 3).Tras lo anterior, se realizó la revisión de contenido en cuanto a la introducción y conclusiones de cada uno de ellos, dando como resultado finalmente 20 artículos, con los cuales se da sustento al tema de investigación del presente documento (Tabla 4).

\section{Análisis de productividad}

En relación al número de publicaciones seleccionadas finalmente y de acuerdo a los criterios de búsqueda, en cuanto al año de publicación, se establecieron dos periodos de cinco años y uno de cuatro años, siendo el periodo comprendido entre el 2009 y el 2013 


\begin{tabular}{|l|c|c|}
\multicolumn{1}{|c|}{ Combinaciones } & $\begin{array}{c}\mathbf{N}^{\circ} \text { de } \\
\text { articulos }\end{array}$ & \% \\
\hline "Higher education" and "disability" & 37 & $31 \%$ \\
\hline "Formation" and "professional" and "disability" & 29 & $24 \%$ \\
\hline "Work formation" and "disability" & 19 & $16 \%$ \\
\hline "Formation" and "technical" and "disability" & 13 & $11 \%$ \\
\hline "Formation" and "technological" and "disability" & 11 & $9 \%$ \\
\hline "Competences" and "technological" and "disability" & 4 & $3 \%$ \\
\hline "Competences" and "professional" and "disability" & 6 & $5 \%$ \\
\hline "Competences" and "technical" and "disability" & 1 & $1 \%$ \\
\hline "Work competences" and "disability" & 1 & $1 \%$ \\
\hline "Formation" and "disability" and "occupational therapy" & 0 & $0 \%$ \\
\hline Total & 121 & $\mathbf{1 0 0} \%$ \\
\hline
\end{tabular}

Tabla 3. Número de publicaciones por base de datos tras el segundo filtro.

\begin{tabular}{|l|c|c|}
\hline \multicolumn{1}{|c|}{ Base de datos } & $\mathbf{N}^{\circ}$ de artículos & $\%$ \\
\hline EBSCO & 23 & $43 \%$ \\
\hline LABORDOC & 19 & $36 \%$ \\
\hline SCIELO & 4 & $8 \%$ \\
\hline LILACS & 7 & $13 \%$ \\
\hline Total & 53 & $100 \%$ \\
\hline
\end{tabular}

Tabla 4. Número de publicaciones por base de datos tras el tercer filtro.

\begin{tabular}{|l|c|c|}
\hline \multicolumn{1}{|c|}{ Base de datos } & $\mathbf{N}^{\circ}$ de artículos & $\%$ \\
\hline LABORDOC & 9 & $45 \%$ \\
\hline EBSCO & 5 & $25 \%$ \\
\hline LILACS & 5 & $25 \%$ \\
\hline SCIELO & 1 & $5 \%$ \\
\hline Total & $\mathbf{2 0}$ & $\mathbf{1 0 0} \%$ \\
\hline
\end{tabular}

el de mayor productividad (Tabla 5). Por otro lado, en lo referente al tipo de estudio e idioma hubo un predominio de las publicaciones de tipo descriptivo y de revisión sistemática (Tabla 6) y de redacción en inglés (Tabla 7).

Tabla 5. Número de publicaciones por periodo de año

\begin{tabular}{|c|c|c|}
\hline Periodo & $\mathbf{N}^{\circ}$ de artículos & $\%$ \\
\hline $2000-2004$ & 8 & $40 \%$ \\
\hline $2005-2008$ & 3 & $15 \%$ \\
\hline $2009-2013$ & 9 & $45 \%$ \\
\hline Total & 20 & $100 \%$ \\
\hline
\end{tabular}

\begin{tabular}{|l|c|c|}
\hline \multicolumn{1}{|c|}{ Tipo de estudio } & $\mathbf{N}^{\circ}$ de artículos & $\%$ \\
\hline Descriptivo & 8 & 40 \\
\hline Revisión sistemática & 8 & 40 \\
\hline Metanalisis & 2 & 10 \\
\hline Guía práctica & 2 & 10 \\
\hline Total & $\mathbf{2 0}$ & $\mathbf{1 0 0} \%$ \\
\hline
\end{tabular}

Tabla 7. Número de artículos por idioma de publicación

\begin{tabular}{|l|}
\hline \multicolumn{1}{|c|}{ Idioma } \\
\hline Inglés \\
\hline Español \\
\hline Total \\
\hline
\end{tabular}

\begin{tabular}{|c|}
\hline $\mathbf{N}^{\circ}$ de artículos \\
\hline 12 \\
\hline 8 \\
\hline 20 \\
\hline
\end{tabular}

\begin{tabular}{|c|}
\hline$\%$ \\
\hline $60 \%$ \\
\hline $40 \%$ \\
\hline $100 \%$ \\
\hline
\end{tabular}

Por último, la clasificación según el continente, Latinoamérica proporciona la mayor cantidad de publicaciones con Colombia, Brasil y Uruguay (Tabla 8) lo cual es de gran ayuda en el trabajo como país respecto al tema de estudio, debido a las características propias del continente.

Tabla 8. Número de artículos por lugar de publicación

\begin{tabular}{|l|c|c|}
\multicolumn{1}{c|}{ Lugar } & $\mathbf{N}^{\circ}$ de artículos & $\%$ \\
\hline Europa & 6 & $30 \%$ \\
\hline Latinoamérica & 7 & $35 \%$ \\
\hline Norteamérica & 3 & $15 \%$ \\
\hline Asia & 2 & $10 \%$ \\
\hline Australia & 2 & $10 \%$ \\
\hline Total & $\mathbf{2 0}$ & $\mathbf{1 0 0} \%$ \\
\hline
\end{tabular}

\section{DISCUSIÓN}

Al establecer un paralelo entre la búsqueda de evidencia y los resultados obtenidos a partir de ella, con la realización del presente análisis bibliométrico, se pone de manifiesto que la discapacidad ha sido una temática estudiada durante los últimos años, aunque existen aspectos que no han sido puestos en la agenda de la investigación formal. Con base en lo anterior, la formación de competencias en la educación superior, sin importar el tipo de discapacidad, es un tema que ha ignorado la legislación existente al respecto en cada uno de los países, especialmente en aquellos de bajos y medianos ingresos.

A la fecha existe escasa evidencia al respecto como se pudo observar en los resultados obtenidos en cuanto al número de publicaciones, las cuales en su contenido no enfatizan en el tipo de formación superior que ofrecen los diversos programas, sino que se generaliza la educación superior.

Lo anterior, podría ser justificado a partir de factores que cada vez son más excluyentes en cada una de las instituciones que pres- 
tan este tipo de formación, especialmente en lo que se refiere a la oferta de programas académicos que sean acorde a los intereses, expectativas y habilidades de cada una de las personas con discapacidad, que deseen acceder a cualquier tipo de formación superior, así como estigmas sociales que ampliamente se han arraigado en la sociedad actual.

Adicional a lo anterior, es importante mencionar que, a pesar de los esfuerzos realizados por las entidades gubernamentales para el acceso de las personas con discapacidad a la formación superior, a través del ajuste de sus sistemas educativos, con el objeto de satisfacer la necesidad de todos los estudiantes, se descuidan aspectos como los ambientes físicos a través de barreras arquitectónicas que hacen aún más difícil la inclusión y el empoderamiento de las personas con discapacidad en la sociedad.

Lo anterior se convierte en un vehículo que genera medios ineficaces para combatir la exclusión, puesto que la inclusión no solo significa integrar a las personas con limitaciones en el sistema educativo, sino también preparar este entorno para recibirlo, lo cual se debe basar en principios como lo son, la aceptación de las diferencias y características individuales, la claridad sobre la condición real de la persona con discapacidad, el valorar a cada persona como un ser único, teniendo en cuenta sus valores, intereses, creencias, destrezas de ejecución, debilidades, y factores que influyen en el desempeño de actividades y restringen su participación en la sociedad, considerando cada uno como un todo y no acciones aisladas como usualmente se evidencia, de tal forma que se permita construir un tipo de sociedad con una misma realidad.

A pesar del notable progreso en el acceso a la educación en relación a décadas pasadas, todavía existe una gran brecha entre las directrices legales existentes y la eficacia en el acceso a la formación superior, puesto que no solo se debe garantizar el ingreso, sino también la permanecía de cada una de las personas con discapacidad en los diversos programas, como se ve evidenciado en las publicaciones de tipo descriptivo a través de encuestas a población con discapacidad que reafirman lo anteriormente dicho. (Molina, 2006).

En una sociedad democrática, con planteamientos educativos universales y de justicia distributiva, no pueden permitirse situaciones en las que algún alumno o alumna, tengan limitada su accesibilidad y desarrollo a los estudios superiores. Desde esos mismos postulados se habrán de diseñar sistemas totales, favorecer estructuras y organización accesibles (recursos, contexto y en actitudes) y desarrollar los mecanismos de compensación, que hagan posible la educación de las personas con discapacidad y su participación en una comunidad inclusiva. (Luque, 2003).

Existe evidencia de la mayoría de las economías occidentales, que las personas con discapacidad tienen menos probabilidades de tener un empleo remunerado y más probable que se les page menos que las personas sin discapacidades. A pesar de la introducción de las dos medidas del lado de la demanda como de la oferta para reducir la brecha salarial y la participación, como la legislación contra la discriminación y la educación y las políticas de formación, la mejora en las últimas décadas ha estado muy por debajo de las metas y expectativas. Los resultados del empleo de personas con discapacidad han mejorado sólo marginalmente y siguen siendo motivo de grave preocupación para la política económica y social por igual. (Consejo Nacional de Política Económica y Social. CONPES, 2014).

Los gobiernos han tratado de hacer frente a la desventaja laboral asociada a la discapacidad mediante la introducción de la legislación contra la discriminación.

Además de las leyes contra la discriminación, la desventaja laboral de las personas con discapacidad se puede abordar a través de la educación y la formación, el objetivo primordial de mejorar la productividad de las personas con discapacidad.

Aunque estudios previos han demostrado que la presencia de las cualificaciones de educación está más fuertemente asociado con el empleo para personas con discapacidad que para las personas sin ningún estudio. El objetivo de este trabajo es examinar el grado para completar el proceso educativo y formación profesional (EFP) ya que esto mejora las tasas de empleo de las personas con discapacidad (Kostas, Cain y Zukunft, 2011).

En relación al acceso laboral las publicaciones permiten determinar la relación directamente proporcional entre la formación y la consecución de un empleo formal, puesto que las ofertas laborales dependen primordialmente de una formación previa que permita la adquisición de experiencia lo cual abre la generación de oportunidades y con ello el desempeño de roles productivos.

\section{CONCLUSIÓN}

Durante la justificación para la realización de este análisis bibliometrico, en relación a lo evidenciado en el quehacer profesional del terapeuta ocupacional en los procesos de rehabilitación e inclusión laboral, se generó una reflexión acerca de la influencia en el acceso a la formación superior de las personas con discapacidad, no solamente en relación a Colombia, sino también a nivel mundial, lo cual permiten concluir que, la formación no solamente básica sino también superior, puede llegar a ser considerada como un elemento básico en los procesos de rehabilitación integral de las personas con cualquier tipo de discapacidad, lo anterior se ha demostrado que influye notablemente en su rehabilitación.

Adicionalmente, los procesos de formación pueden generar impacto positivo en la interacción social, en la mejora de la autoestima, en las habilidades sociales, autocuidado, autonomía y redes de apoyo, que permiten que la persona se incluya y empodere en la sociedad, siendo menos vulnerable a la exclusión tanto social como laboral.

Aunque el marco legislativo en discapacidad, pone en manifiesto la inclusión y equidad, la distancia entre la normatividad y la realidad presenta vacíos, especialmente en lo que se refiere a la ejecución e implementación de los programas y el empoderamiento de cada uno de los actores, es decir, que no solo se debe garantizar el acceso, sino también la calidad de lo que allí se promulga, de tal forma que se adquieran competencias mínimas de acuerdo al enfoque de formación, que permitan mejoras en el acceso laboral y permanencia, 
lo cual esta intrínsecamente relacionado con la calidad de vida y el desempeño ocupacional de cada persona.

Lograr en la práctica lo planteado idealmente no es un asunto fácil, debido a que implica responsabilidades no solo del estado, sino también de las personas con discapacidad, sus familias, la comunidad, las instituciones y la sociedad en general, es decir, exige el compromiso de todos para poder conseguir una sociedad inclusiva, cuyos principios fundamentales sean la equidad y la calidad.

La Inclusión de las Personas con Discapacidad en la Formación Profesional es un gran esfuerzo de promoción para promover la formación de las personas en condición de discapacidad en conjunto con sus compañeros no discapacitados. Durante décadas, la OIT ha promovido la igualdad de trato y la igualdad de oportunidades de las personas con discapacidad, incluidos en los programas de desarrollo de habilidades y la empleabilidad.

Con la adopción y la tasa de ratificación notable de la Convención de la ONU sobre los Derechos de las Personas con Discapacidad a la formación y el empleo está atrayendo renovada atención. Los países están modificando las leyes para garantizar estos derechos $\mathrm{y}$, volviéndose hacia sus sistemas de formación para garantizar que las personas con discapacidad puedan participar activamente de dicho proceso. (International Labour Office, 2013).

El camino por recorrer es todavía muy largo, se deben generar planes, proyectos y programas en conjunto a partir de la diversidad funcional, por lo que es de gran importancia la participación no solamente de profesiones como la terapia ocupacional desde su objeto de estudio que es el desempeño ocupacional y los factores que influyen en ello, sino también la participación activa de todos como sociedad, por lo que se hace extensiva la invitación, no solamente a las profesiones del área de la salud y sociales, sino a todas en general, a investigar y publicar en relación a la temática, desde cada uno de los enfoques de formación.

La participación en la vida corriente de la persona con discapacidad debe ser deacuerdo a las posibilidades de preparación y formación adecuadas y la reducción o eliminación de obstáculos sociales, jurídicos o arquitectónicos y de otra naturaleza. Se concluye entonces que para lograr la integración de la persona con discapacidad a la vida productiva se requiere que ésta quiera hacerlo y que se prepare adecuadamente, no solo en el oficio acorde a sus capacidades, sino que también aprenda a enfrentar lo que esto implica. (Machado, 2005). 


\section{REFERENCIAS}

Consejo Nacional de Política Económica y Social. CONPES 166. Recuperado el 11 de Abril de 2014, de: http://www.colombiaaprende.edu.co/ html/micrositios/1752/articles-335918_archivo_pdf.pdf

Departamento Nacional de Estadística DANE. (2005) Discapacidad: Informe estadístico. [Consultado el 1 de abril de 2014]. Disponible en: http://www.dane.gov.co/index.php/poblacion-y-registros-vitales/discapacidad

Discapacidad Colombia. (sf). El Banco Mundial y la discapacidad. [Consultado el 1 de abril de 2014].Disponible en: http://discapacidadcolombia.com/modules.php?name=Content\&pa=showpage\&pid=35

International Labour Office.(2006).Strategies for skills acquisition and work for people with disabilities . Geneva. Recuperado de http:// www.ilo.org/wcmsp5/groups/public/@ed_emp/@ifp_skills/documents/publication/wcms_107784.pdf

International Labour Office.(2013).Inclusion of people with disabilities in vocational training : a practical guide. Geneva. Recuperado de http://www.ilo.org/wcmsp5/groups/public/---dgreports/-gender/documents/publication/wcms_230732.pdf

Kostas, M., Cain, P. \& Zukunft, A. (2011). Improving the employment rates of people with disabilities through vocational education. Discussion Paper, 5548, 1-35
Luque, D. J. (2003). “Trastornos del desarrollo, discapacidad y necesidades educativas especiales: Elementos psicoeducativos”. Revista Iberoamericana de Educación. http://www.campus-oei.org/ revista/deloslectores/372Luque.PDF.

Machado, C. F.(200o) Guía para la recuperación de experiencias en procesos de integración normalizada de personas con discapacidad en la formación profesional integral. Montevideo Recuperado de http://www.oitcinterfor.org/sites/default/files/file_publicacion/recexpint.pdf.

Molina, R. (2006), Hacia una educación con igualdad de oportunidades para personas con discapacidad. Revista de la Facultad de Medicina de la Universidad Nacional, 54(2),148-154.

Organización Mundial de la Salud. (2011). Informe Mundial de la Discapacidad: OMS.

Servicio Nacional de Aprendizaje. (sf). Servicio al ciudadano: Glosario. [Consultado el 26de febrero de 2014]. Disponible en: http://mgiportal.sena.edu.co/Portal/Servicio+al+Ciudadano/Glosario/

Senado de la República de Colombia. (1991).Constitución Política de Colombia. Colombia.

REV. COL. REH 2014 || Volumen 13 || Páginas 88 - 95 || 